\title{
The fusion protein of peste des petits ruminants virus is a hemolysin
}

\author{
Brief Report \\ L. R. Devireddy ${ }^{1, *}$, R. $\operatorname{Raghavan}^{1}$, S. Ramachandran $^{2}$, and M. S. Shaila ${ }^{3}$ \\ ${ }^{1}$ Department of Veterinary Microbiology, Veterinary College, Bangalore, India \\ ${ }^{2}$ Center for Tropical Veterinary Medicine, The University of Edinburgh, \\ Roslin, Midlothian, Scotland, U.K. \\ ${ }^{3}$ Department of Microbiology and Cell Biology, Indian Institute of Science, \\ Bangalore, India
}

Accepted December 31, 1998

Summary. The fusion glycoprotein (F protein) of paramyxoviruses plays a vital role in virus-induced cytopathology. To explore the role of the F protein in peste des petits ruminants virus (PPRV)-induced cytopathology, the F protein of PPRV was purified by immunoaffinity chromatography. The purified $\mathrm{F}$ protein, when incubated with chicken erythrocytes, caused lysis suggesting that PPRV F protein is a hemolysin. Furthermore, the hemolysis can be inhibited by hyperimmune serum against $F$ protein. The virus-induced cell fusion (syncytia) was also inhibited by the hyperimmune serum against the F protein. In summary, these results indicate that the purified PPRV F protein is biologically active and is involved in virus-induced hemolysis, cell-fusion and the initiation of infection.

Peste des petits ruminants (PPR) is a disease of small ruminants caused by a virus that is serologically related to rinderpest virus (RPV). The disease is characterized by necrotizing enteritis and erosive stomatitis, enteritis and pneumonia (for review see [10]). Furthermore, the morbidity and mortality rates are high. Thus, this disease is a serious problem for small ruminant industry. PPRV was classified as a number of the morbillivirus genus of the paramyxovirus family [6]. Subsequent

*Present address: Department of Veterinary and Biomedical Sciences, University of Nebraska-Lincoln, East Campus, Lincoln, NE, U.S.A. 
studies have shown that PPRV is distinct from RPV based on protein size and DNA probes [5].

The envelope of morbilliviruses posesses several biological activities which include hemagglutination, receptor binding, neuraminidase, hemolysing, and cellfusing activities. The glycoprotein spikes on the envelope are important in facilitating adsorption and penetration of the virus particle into the susceptible cell. The two surface glycoproteins, Hemagglutinin $(\mathrm{H})$ and Fusion $(\mathrm{F})$ proteins are therefore important for the initiation and the progression of the infection (reviewed in [17]). The $\mathrm{H}$ protein determines the host-specificity because it recognizes receptors on the cell membrane and anchors the virus to the receptors. Antibody against the $\mathrm{H}$ protein prevents virus attachment. The $\mathrm{F}$ protein fuses the viral envelope to the cell membrane thereby releasing the viral nucleocapsid into the cell [2]. The $\mathrm{H}$ and $\mathrm{F}$ glycoproteins of PPRV have been purified in our earlier studies [4]. Immunization of rabbits with purified $\mathrm{H}$ or $\mathrm{F}$ proteins PPRV protected against a lethal challenge equally well [4]. Therefore, antibody response to either $\mathrm{H}$ or $\mathrm{F}$ protein may be sufficient to confer protection.

Except for measles virus (MV), PPRV is the only morbillivirus which causes hemagglutination of red blood cells (RBC). Although much is known about PPRV $\mathrm{H}$ protein, studies of the F glycoprotein of PPRV are scarce; however, data on $\mathrm{F}$ protein of other paramyxoviruses indicate that it is synthesized as a biologically inactive precursor $\left(\mathrm{F}_{0}\right)$ which has a molecular weight $(\mathrm{Mr})$ of $65 \mathrm{kDa}$. This $\mathrm{F}_{0}$ moiety is cleaved by a host-cell protease to yield two polypeptides ( $\mathrm{F}_{1}$ and $\mathrm{F}_{2}$ ) with Mrs of 50 and $15 \mathrm{kDa}$. The F protein, in its biologically active form, is responsible for important biological activities, such as penetration of the virus into the host cell through the fusion of viral and cell membranes, cell fusion, and hemolysis (reviewed in [3], [18], [20]). Purified F protein has no biological activity; however, when reconstituted into a membrane with lipids, it is biologically active. This suggests that $\mathrm{F}$ protein requires attachment to the cell to exert its effects [9]. This further suggests that the activities of the $F$ protein involve the fusion of membranes. Sendai virus mutants, containing the uncleaved $\mathrm{F}_{0}$ precursor, were not infectious and also lacked hemolyzing and cell-fusion activities $[8,20]$. Similarly, the virulence of Newcastle disease virus was also shown to be correlated with the ability of the host cell to cleave the viral $F_{0}$ polypeptide [11]. Thus, all of these studies indicate that the $F$ protein plays a central role in the pathogenesis of paramyxovirus by influencing tropism and the ability of the virus to undergo multiple replication, to spread in the host, and thus to cause disease.

PPRV is distinct from other paramyxoviruses in many aspects. For example, PPRV $\mathrm{H}$ protein agglutinates $\mathrm{RBC}$ whereas, $\mathrm{H}$ protein of the majority of the paramyxoviruses, does not cause hemagglutination. Although it is known that paramyxovirus $\mathrm{F}$ protein causes hemolysis, it is not known if PPRV F protein has the same property. In this study, we explored the biological activities of the F protein of PPRV. Our results suggest that purified PPRV F protein causes lysis of chicken RBC. Antibodies against F protein not only inhibited hemolysis but also PPRV-induced cell fusion. 
The F protein of Indian strain of PPRV (TN87/1 [21]) was purified from infected Vero cell lysates in immunoaffinity chromatography using antibody against the amino-terminus of the $\mathrm{F}_{1}$ protein of MV (a gift from T. Barrett, Institute of Animal Health, Pirbright, UK) as described in Varasanyi et al. [22]. Briefly, the IgG fraction of rabbit polyclonal antibody against $F_{1}$ protein of $\mathrm{MV}$ was coupled to cyanogen bromide activated Sepharose- $4 \mathrm{~b}$ (Pharmacia). This affinity column was mixed with infected cell extracts and held on ice for two hours with agitation. The matrix was then washed with TEN buffer $(10 \mathrm{mM}$ Tris-HCl, $\mathrm{pH} 8.0 ; 1 \mathrm{mM}$ EDTA and $0.1 \%$ NP-40). The F protein was desorbed with 3M KSCN in TEN buffer. One cycle of adsorption and desorption produced a highly purified preparation of the $\mathrm{F}$ protein. The protein content was measured by the method of Bradford [1]. The homogeneity of the purified protein was established in SDS-PAGE followed by silver staining (data not shown). To further confirm the identity of the F protein Western blot was done using the antibody against the amino-terminus of the $\mathrm{F}_{1}$ protein of MV as described in Harlow and Lane [7]. Only anti- $\mathrm{F}_{1}$ antibody but not PPRV anti-H antibody detected PPRV F protein (data not shown). The estimated molecular weight of the F protein was $46 \mathrm{kDa}$. Therefore, these results suggest that PPRV F protein is purified to homogeneity.

The purified $\mathrm{F}$ protein was used as an antigen to raise the hyperimmune serum in rabbits. Serum samples were collected at 4, 14, 15, 16 and 20 weeks after immunization. Antibody response to F protein was evaluated in ELISA using purified virus as an antigen. A high titered antibody response titer to the F protein was observed suggesting that purified $\mathrm{F}$ protein retained immunogenicity.

It is known that the F protein of paramyxoviruses cause lysis of erythrocytes [19]. Further, in this study we explored the hemolysis activity of the purified $F$ protein of PPRV. Hemolysis (HL) assay was described in Norrby and Gollmar [12]. Specified amounts of protein was mixed with $10 \%$ chicken erythrocytes and incubated at $30^{\circ} \mathrm{C}$ for $30 \mathrm{~min}$. The absorbance of the supernatant was measured at $540 \mathrm{~nm}$. Purified F protein specifically lysed the chicken RBC. No hemolysis was observed when purified $\mathrm{H}$ protein was incubated with chicken RBC. Addition of $1.5 \mu \mathrm{g}$ of purified $\mathrm{H}$ protein also did not increase hemolysis activity of the F protein (Fig. 1). Therefore, these results indicate that the F protein alone is sufficient to cause lysis of RBC. Furthermore, incubation of purified virus with chicken $\mathrm{RBC}$ also caused lysis albeit to a lower extent (data not shown). Interestingly, the hemolysis activity of the F protein is dose-dependent. An increase in the amount of $F$ protein caused an increase in the lysis of RBC (Fig. 1). No lysis was observed when RBC were incubated with bovine serum albumin (BSA) (Fig. 1). Taken together, these results suggest that purified PPRV F protein is a hemolysin.

To demonstrate the specificity of the hemolysis activity of PPRV F protein, hemolysis inhibition (HLI) was done. The purified $\mathrm{F}$ protein was incubated with antibodies against the amino-terminus of the $\mathrm{F}_{1}$ protein of MV or rabbit hyperimmune sera against PPRV F protein. Both antibodies completely neutralized the hemolysis activity of PPRV F protein (data not shown). Incubation of $F$ protein with normal rabbit serum or anti-H hyperimmune serum did not neutralize 


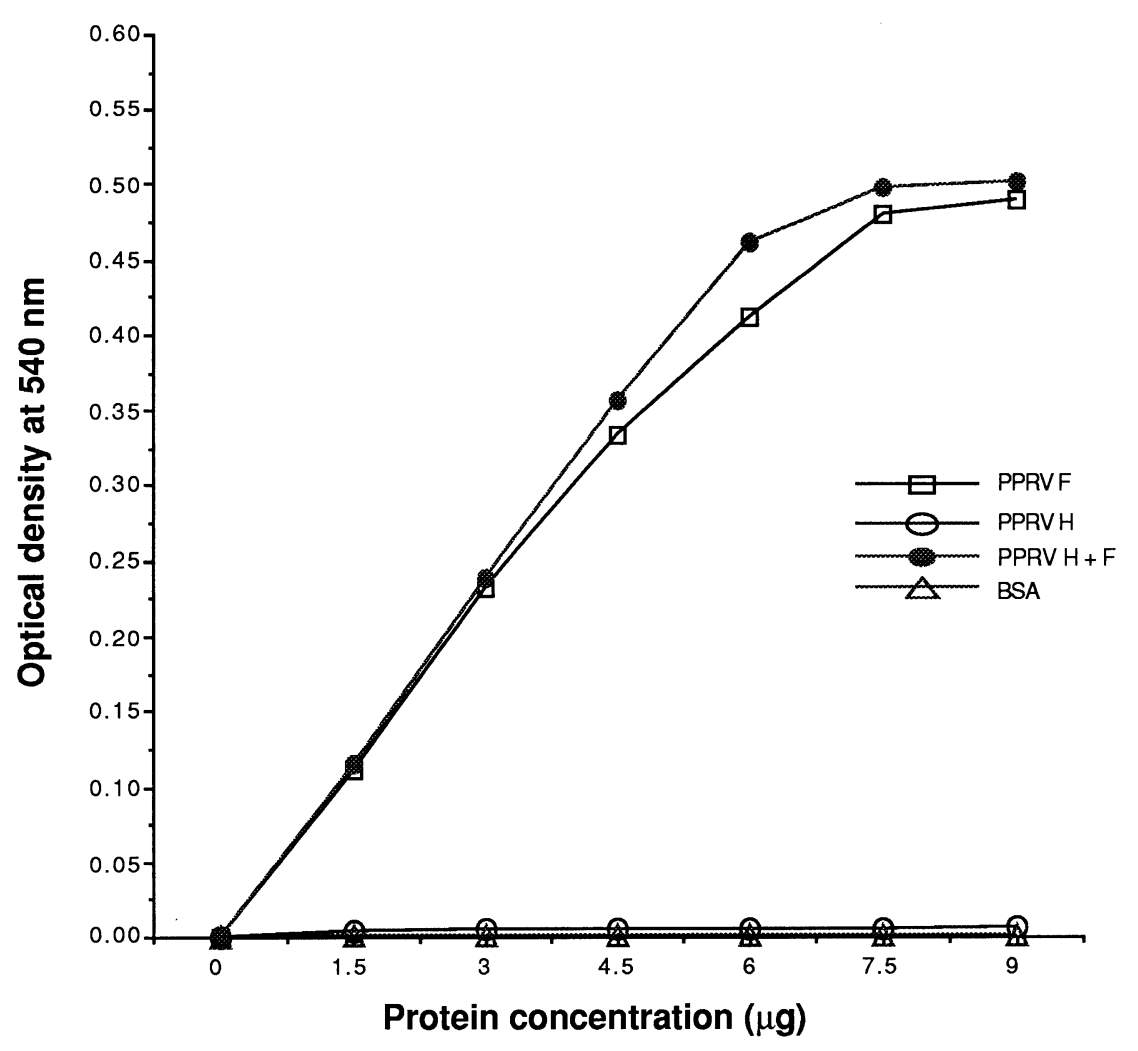

Fig. 1. Hemolysis by purified PPRV F protein. Chicken RBC were incubated with indicated proteins at different concentrations. Hemolytic activity of the F protein is expressed as absorbance reading at $540 \mathrm{~nm}$

hemolysis activity of PPRV F protein. Thus these results suggest that antibodies against PPRV F protein can inhibit hemolysis by PPRV F protein.

Paramyxoviruses spread from one cell to another cell by inducing syncytia. The $\mathrm{F}$ protein plays a viral role in inducing syncytia formation. In this study we assayed the ability of antibodies to $\mathrm{F}$ protein to inhibit the spread of infection from cell to cell. Fusion-inhibition assays were as described in Ray et al. [16]. Confluent monolayers of baby hamster kidney (BHK 21) cells were grown on cover slips and infected with virus at high multiplicity of infection (m.o.i.). These conditions mimic more closely the conditions in the animal whose respiratory or oral epithelium has been seeded with an inoculum of virus. After $12 \mathrm{~h}$ of infection, cells were washed and fresh medium containing 1 in 500 dilutions of preimmune serum or test sera was added. After 36 hours of addition of sera, cells were stained with Giemsa. PPRV-induced syncytia in infected baby hamster kidney (BHK21) cells (Fig. 2A). The hyperimmune serum against PPRV F protein completely inhibited the formation of syncytia (Fig. 2B). However, antibody against $\mathrm{F}_{1}$ protein of MV partially inhibited the formation of syncytia (Fig. 2C). On the contrary, normal rabbit serum did not inhibit the formation of syncytia (Fig. 2D). These results suggest that anti-F antibodies inhibited the syncytia formation. 

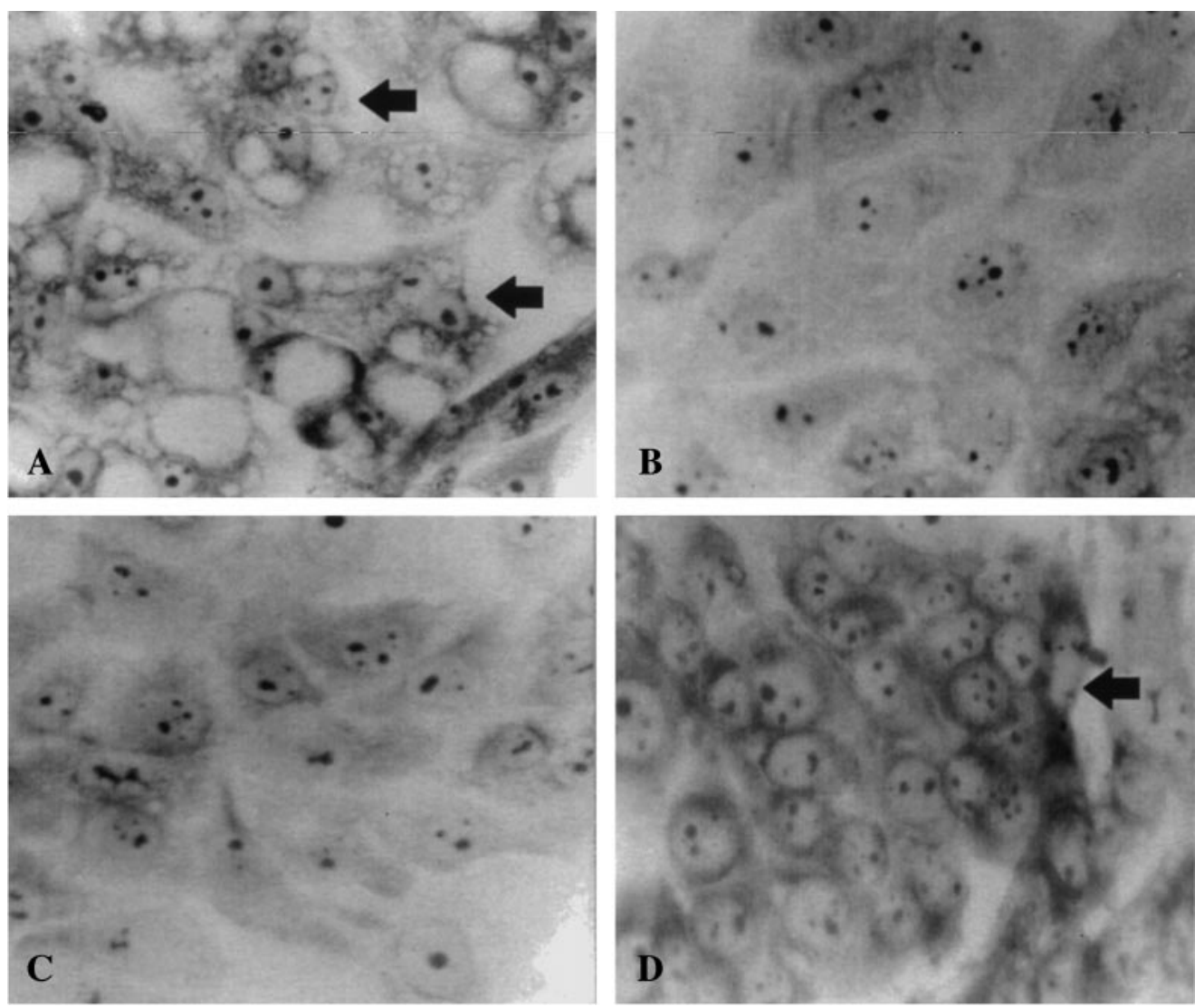

Fig. 2. Fusion inhibition by PPRV F antibody in PPRV-infected BHK21 cells. A Untreated infected cells showing characteristic syncytia; B infected cells treated with PPRV F antibody obtained 14 weeks after immunization; $\mathbf{C}$ infected cells treated with antibody against the amino terminus of the $\mathrm{F}_{1}$ protien of $\mathrm{MV} ; \mathbf{D}$ infected cells treated with normal rabbit serum

It has long been known that the surface glycoproteins of enveloped viruses are involved in the generation of immunity to infection. However, the determination of the importance of the individual paramyxovirus glycoproteins required the use of specific antibodies to each protein and an examination of the effectiveness of these antibodies in preventing not only the initiation of infection but also the spread of infection. The importance of a proper immune reponse to the F protein for the induction of protective immunity has been documented extensively for several members of paramyxovirus family [2, 13-15]. The incomplete protective response in animals immunized with either formalin-inactivated virus or with lower doses of envelope glycoproteins could be because of the lack of antibody response to the F protein [16]. In our previous studies we have demonstrated that immunization of rabbits with purified PPRV F protein protected rabbits against a lethal challenge, proving that anti-F antibodies are protective [4].

It is intriguing to note that purified PPRV causes hemolysis. The observation that purified $\mathrm{F}$ protein but not the $\mathrm{H}$ protein causes lysis of $\mathrm{RBC}$ suggests that 
F protein confers hemolytic property to the PPRV. This hypothesis is further supported by the fact that antibodies against $\mathrm{F}$ protein not to the $\mathrm{H}$ protein inhibited hemolytic activity. Other members of the paramyxovirus family that were shown to have both hemagglutination and hemolysis activities are SV5 virus and MV [12, 19]. The observations that PPRV not only agglutinates RBC but also lyses them suggest that PPRV is more related to MV than RPV in the current morbillivirus pentad.

In a population of cells, paramyxoviruses can spread either by released virus adsorbing to and infecting other cells or by fusion of the membrane of an infected cell with that of an adjacent cells as a result of the activity of the F protein. Interestingly, the antibodies against $\mathrm{H}$ protein prevented the spread of infection by released virus but they did not prevent the spread of infection by membrane fusion [15]. In contrast, antibodies to the F protein completely prevented the spread of infection because they were capable not only of neutralizing released virus but also of inhibiting the activity of the $\mathrm{F}$ protein and thereby preventing cell to cell spread by membrane fusion. Thus, in cells that were susceptible to the fusing activities of the $F$ protein, infection spread from cell to cell even in the presence of antibodies to $\mathrm{H}$ protein. Results presented here also suggest that antibodies against the PPRV F protein inhibited PPRV-induced cell-fusion. Thus, these results further corroborate the hypothesis that effective immunological prevention of the spread of paramyxovirus infection must involve neutralization of the $F$ protein and thus an effective vaccine must induce antibodies to this protein.

\section{Acknowledgements}

This work was a part of the M. V. Sci. thesis of LRD. LRD was supported a fellowship from the Indian Council of Agricultural Research (ICAR). We thank Dr. V.V.S. Suryanarayana for his suggestions, Dr. Pande for collecting chicken erythrocytes, Dr. Hegde for animal experimentation and Dr. Sarathi for his help in microscopy. We also thank Judi Galeota and Doreen Bailey for their help in the preparation of the manuscript.

\section{References}

1. Bradford MM (1976) A rapid and sensitive method for the quantitation of microgram quantities of protein utilizing the principle of protein-dye binding. Anal Biochem 72: 248-254

2. Choppin PW, Scheid A (1980) The role of viral glycoprotein in adsorption, penetration and pathogenecity of viruses. Rev Infect Dis 2: 40-61

3. Choppin PW, Richardson CD, Merz DC, Hall WW, Scheid A (1981) The function and inhibition of membrane glycoproteins of paramyxoviruses and myxoviruses and the role of measles virus $M$ protein in subacute sclerosing panencephalitis. J Infect Dis 143: 352-363

4. Devireddy LR, Raghavan R, Ramachandran S, Subbarao MS (1998) Protection of rabbits against lapinized rinderpest virus using purified envelope glycoproteins of peste des petits ruminants and sheep rinderpest viruses. Acta Virol 42: 299-306

5. Diallo A, Barrett T, Lefevere P-C, Taylor WP (1987) Comparison of proteins induced in cells infected with rinderpest and peste des petits ruminants viruses. J Gen Virol 68: $2033-2038$ 
6. Gibbs EPJ, Taylor WP, Lawman MPJ, Bryant J (1979) Classification of peste des petits ruminants virus as the fourth member of the genus morbillivirus. Inter Virol 11:268-274

7. Harlow E, Lane DP (1988) Immunoblotting. In: Harlow E, Lane DP (eds) Antibodies: A laboratory manual. CSH Press, Cold Spring Harbor, pp 471-510

8. Homma M, Ohuchi M (1973) Trypsin action on the growth of sendai virus in tissue culture cells III. Structural differences of sendai viruses growth in eggs and tissue culture cells. J Virol 12: 1457-1 465

9. Hsu M-C, Scheid A, Choppin PW (1979) Reconstitution of membranes with individual paramyxovirus glycoproteins and phospholipid in cholate solution. Virology 95: 476491

10. Lefevre P-C, Diallo A (1990) Peste des petits ruminants. Rev Sci Tech Off Int Epiz 9: 951-965

11. Nagai Y, Klenk H-D (1977) Activation of precursors to both glycoproteins of Newcastle disease virus by proteolysis cleavage. Virology 77: 125-134

12. Norrby E, Gollmar Y (1975) Identification of measles virus-specific hemolysis-inhibiting antibodies separate from hemagglutination-inhibiting antibodies. Infect Immun 11:231239

13. Norrby E, Enders-Ruckle G, Termeulen V (1975) Differences in the appearance of antibodies to structural components of measles virus after immunization with inactivated and live virus. J Infect Dis 132: 262-269

14. Norrby E, Utter G, Orvell C, Appel M (1986) Protection against canine distemper virus in dogs after immunization with isolated fusion protein. J Virol 58: 536-541

15. Merz DC, Scheid A, Choppin PW (1980) Importance of antibodies to the fusion glycoprotein of paramyxovirus in the prevention of spread of infection. J Exp Med 151: 275-288

16. Ray R, Brown UE, Compans RW (1985) Glycoproteins of human parainfluenza virus type 3. Characterization and evaluation as a subunit vaccine. J Infect Dis 152: 1219-1 230

17. Rima BK (1983) The proteins of morbilliviruses. J Gen Virol 64: 1 205-1 219

18. Scheid A, Caliguiri LA, Compans RW, Choppin PW (1972) Isolation of paramyxovirus glycoprotein. Association of both hemagglutinating and neuraminidase activities with the larger SV5 glycoprotein. Virology 50: 640-652

19. Scheid A, Choppin PW (1974) Identification of the biological activities of paramyxovirus glycoproteins. Activation of cell fusion, hemolysis and infectivity by proteolytic cleavage of an inactive precursor protein of Sendai Virus. Virology 57: 475-490

20. Scheid A, Choppin PW (1977) Two disulfide-linked polypeptide chain constitutes the active $F$ protein of paramyxoviruses. Virology 80: 54-66

21. Shaila MS, Venugopal K, Purusothaman V, Venkateshan RA (1990) Isolation and identification of peste des petits ruminants virus from an outbreak of rinderpest-like disease in Tamilnadu sheep. Ind Vet J 67: 383-384

22. Varasanyi TM, Utter G, Norrby E (1984) Purification, morphology and antigenic characterization of measles virus envelope components. J Gen Virol 65: 355-366

Authors' address: Dr. L. R. Devireddy, Department of Veterinary and Biomedical Sciences, Room 103, University of Nebraska-Lincoln, East Campus, Lincoln, NE 68583-0905, U.S.A. 